\title{
Criminologie
}

\section{La négociation des plaidoyers de culpabilité : une pratique hétérogène}

\section{Sylvie Gravel}

Volume 24, numéro 2, 1991

Regard sur les tribunaux

URI : https://id.erudit.org/iderudit/017307ar

DOI : https://doi.org/10.7202/017307ar

Aller au sommaire du numéro

\section{Éditeur(s)}

Les Presses de l'Université de Montréal

ISSN

0316-0041 (imprimé)

1492-1367 (numérique)

Découvrir la revue

Citer cet article

Gravel, S. (1991). La négociation des plaidoyers de culpabilité : une pratique hétérogène. Criminologie, 24(2), 5-29. https://doi.org/10.7202/017307ar
Résumé de l'article

This article presents the principal results of a study on plea bargaining at the Montreal Justice Hall. Various methods were adopted: observations, a thorough study of the files, interviews and analyses of the judicial statistics. The results show that negotiations vary considerably depending on the gravity of the offence concerned. In the case of a minor charge or a murder charge, the characteristics as well as the stakes and consequences of the plea bargaining are very different. In minor cases, the negotiations are reduced to their simplest form. They are a routine process of cooperation between the parties rather than bargaining to get an admission of guilt by promising the accused a reduced charge or sentence. On the contrary, the negotiations in murder cases take the traditional form of plea bargaining in which the power struggle is dominant. The study shows that it is in cases of murder than plea bargaining presents most difficulties. 


\section{LA NÉGOCIATION DES PLAIDOYERS DE CULPABILITÉ: UNE PRATIQUE HÉTÉROGÈNE}

Sylvie Gravel ${ }^{1}$

This article presents the principal results of a study on plea bargaining at the Montreal Justice Hall. Various methods were adopted: observations, a thorough study of the files, interviews and analyses of the judicial statistics. The results show that negotiations vary considerably depending on the gravity of the offence concerned. In the case of a minor charge or a murder charge, the characteristics as well as the stakes and consequences of the plea bargaining are very different. In minor cases, the negotiations are reduced to their simplest form. They are a routine process of cooperation between the parties rather than bargaining to get an admission of guilt by promising the accused a reduced charge or sentence. On the contrary, the negotiations in murder cases take the traditional form of plea bargaining in which the power struggle is dominant. The study shows that it is in cases of murder than plea bargaining presents most difficulties.

En Amérique du Nord, la négociation des plaidoyers de culpabilité est sûrement, après la disparité des sentences, l'un des sujets les plus discutés en matière d'administration de la justice. Depuis les premiers articles américains à la fin des années 1960, les écrits sur le thème de la négociation de plaidoyer, communément appelée plea bargaining par les Anglo-Saxons, se sont en effet multipliés et diversifiés. On dénombre aujourd hui une quantité impressionnante de commentaires, réflexions, prises de position et études sur le sujet.

Toutefois, curieusement, la problématique de la négociation de plaidoyer n'a pas suscité au Canada autant d'intérêt que chez nos voisins américains. Les écrits canadiens sont en effet relativement peu nombreux et les études empiriques extrêmement rares ${ }^{2}$. Le peu d'informations disponibles sur l'étendue, la nature et les conséquences de la négociation de plaidoyer au Canada a été déploré par plusieurs observateurs (Commission canadienne sur la détermination de la peine, 1987; Cousineau et Verdun-Jones, 1979; Ferguson et

1. L'auteure est agente de recherche au Centre international de criminologie comparee de l'Université de Montréal, C.P. 6128, Succ. A, Montréal (Québec) H3C 357.

2. Voir pour le Canada anglais les études de Grosman (1969), Hartnagel (1975), Klein (1976) et Ericson et Baranek (1982). Au Québec, Poirier (1986), s'intéressant aux pratiques de travail des avocats de la défense dans l'organisation pénale, fut, à notre connaissance, le premier à recueillis des données empiriques sur le phénomène de la négociation de plaidoyer. 
Roberts, 1974). Or, l'absence de données empiriques influence indiscutablement la qualité du débat concernant la légitimité de cette pratique. On constate en effet que ses opposants et ses défenseurs fondent souvent leur argumentation sur des opinions qui ne sont pas nécessairement supportées par les faits.

Dans le but de combler, en partie du moins, ce besoin urgent de données empiriques québécoises et canadiennes sur le phénomène de la négociation de plaidoyer, une étude a été entreprise au Centre international de criminologie comparée de l'Université de Montréal (Gravel et Baril, 1990). Dans le cadre de cet article, nous discuterons de l'un des principaux résultats de la recherche: l'identification de deux formes particulières de négociation ayant des caractéristiques, des enjeux et des conséquences distincts. L'étude confirme en effet que la négociation de plaidoyer ne constitue pas une pratique uniforme: nous avons découvert deux façons bien différentes de négocier selon la gravité des accusations portées. Mais avant d'en discuter plus à fond, quelques mots, d'abord, au sujet de la méthodologie adoptée.

\section{L'APPROCHE MÉTHODOLOGIQUE}

La négociation de plaidoyer est un processus informel: elle se déroule à huis clos, sans autre témoin que les parties impliquées. Ce caractère occuite de la pratique fait en sorte que les informations sur le sujet sont difficilement accessibles. Afin de pallier cette difficulté inhérente à notre objet d'étude, il nous est apparu préférable de privilégier la diversité des techniques de cueillette de données, dans le but d'obtenir la plus grande quantité d'informations possible.

Deux méthodes de cueillette de données ont constitué nos sources primaires d'informations: l'observation en cour et l'étude spécifique de dossiers de meurtre ayant fait l'objet d'une négociation de plaidoyer. Elles ont été complétées par des entretiens menés auprès d'avocats de la défense, de procureurs de la Couronne et de juges et par l'analyse des statistiques judiciaires. Enfin, des entrevues avec des victimes et des accusés dont les causes se sont réglées par une négociation de plaidoyer ont également été effectuées.

Afin de bien comprendre en quoi consiste la négociation de plaidoyer, nous avons donc choisi de l'observer «de l'intérieur», c'est-à-dire d'être le témoin privilégié des interactions entre les procureurs de la Couronne et les avocats de la défense et, éventuellement, tout autre participant à la négociation. Nous avons eu l'occasion d'assister aux négociations entre les procureurs de la Couronne et de la défense, qu'elles aient eu lieu en cour, dans des cubjcules attenants aux salles d'audience ou, dans certains cas, dans le bureau du procureur de la Couronne. Nous avons été témoin de 68 négociations sur 
une période de 18 jours d'observation. Les observations ont été effectuées dans les salles d'audience où sont entendues quotidiennement une dizaine, une vingtaine et même une centaine de causes et que l'on nomme dans le jargon «salles à volume». Un intérêt particulier a été porté à la chambre de pratique, communément nommée le «406», du numéro de la salle où se concentrent ces activités, qui traite une centaine de dossiers journellement, la plupart s'y retrouvant afin qu'un plaidoyer de culpabilité de la part de l'accusé soit enregistré.

Les négociations que nous avons observées concernent avant tout les délits mineurs. Pour des motifs qu'il serait trop long d'exposer ici, la stratégie d'observation adoptée ne permettait pas de recueillir des informations concernant la nature des négociations qui ont cours lorsqu'il s'agit de délits plus graves. Nous avons résolu ce problème par l'étude en profondeur de 15 dossiers d'homicide ${ }^{3}$ pour lesquels les deux avocats en cause, soit ceux de la défense et de la poursuite, ont été rencontrés et interrogés sur les raisons qui les avaient incités à s'engager dans une négociation, sur le déroulement des discussions et, éventuellement, sur l'entente qui avait été conclue.

L'univers de l'étude a été restreint au palais de justice de Montréal. Aux fins de la recherche, la négociation de plaidoyer a été définie de la façon suivante: une pratique selon laquelle la poursuite et la défense négocient entre elles la détermination d'une affaire pénale ${ }^{4}$.

\section{LES NÉGOCIATIONS DANS LES AFFAIRES MINEURES}

Les délits mineurs pour lesquels nous avons assisté à une négociation sont très majoritairement ceux contre les biens : vol, introduction par effraction, fraude, méfait. Les délits sans victime relatifs à la possession et au trafic de stupéfiants, à la conduite d'un véhicule moteur avec les facultés affaiblies et au bris de condition, bien que d'une incidence moindre, sont également nombreux. On ne retrouve que quelques dêlits contre la personne: le plus souvent il s'agit de voies de fait et d'agressions sexuelles simples.

\section{LE DÉROULEMENT DES NÉGOCIATIONS}

Habituellement, les négociations se tiennent dans des cubicules à l'extérieur de la salle d'audience. Mais elles peuvent aussi avoir lieu à l'intérieur

3. Le choix de l'homicide plutôt que toute autre accusation a été motivé par la problématique particulière soulevée en entrevues par les juristes mais surtout par les victimes et les accusés que ce type de délit présente au regard de la négociation de plaidoyer.

4. Nous avons repris la définition énoncée par le Barreau du Québec (1987). 
même de la salle de cour, soit avant que les audiences ne débutent, pendant le déroulement de celles-ci ou lors d'une pause. Plus rarement, l'avocat de la défense rencontre le procureur de la Couronne à son bureau ou lui téléphone le matin même ou quelques jours avant la date prévue de l'audience.

Les victimes et les accusés sont totalement exclus des discussions. Les policiers enquêteurs, eux, n'assistent que très rarement aux négociations, et lorsqu'ils sont présents, ils ne prennent pas une part active aux pourpariers. Le plus souvent, leur rôle consiste à préciser les faits de l'affaire. Quant aux juges, bien que la majorité ne participent pas aux discussions, certains d'entre eux sont consultés et informent les avocats de la sentence qu'ils imposeraient à l'accusé dans l'éventualité où ce dernier décidait de reconnaître sa culpabilité.

Ce qui étonne au sujet des négociations qui interviennent dans les dossiers mineurs, c'est leur brièveté: quelques minutes seulement, voire quelques secondes, suffisent aux deux parties pour en arriver à un accord. Les ententes se concluent rapidement en raison du peu de controverse qui entoure les discussions. Le modèle de négociation le plus répandu est celui où, après un examen sommaire des circonstances du délit, une des parties fait une proposition de sentence, généralement le procureur de la Couronne, et l'autre partie l'accepte. C'est la façon la plus commune de régler les dossiers. En voici deux exemples.

(1) (Introduction par effraction, possession illégale de biens volés ${ }^{5}$ )

A.D. ${ }^{6}$ : Il va plaider coupable, il n'a pas le choix. Je te suggère une sentence suspendue.

P.C.: O.K.

(2) (Vol de moins de mille dollars, possession illégale de biens volés)

A.D.: Il n'a pas d'antécédents. Il a perdu son emploi à la suite du vol. Je plaiderais à recel.

P.C.: O.K.

A.D.: Qu'est-ce que tu veux? Une amende?

P.C.: Une amende de trois cents dollars.

A.D.: O.K.

5. Énumération des accusations auxquelles l'accusé faisait face.

6. Les sigles «A.D.» et «P.C.» signifient respectivement «avocat de la défense» et "procureur de la Couronne». 
En certaines occasions, la proposition faite par l'une des parties ne concerne que la nature de la peine: les avocats s'entendent pour suggérer au juge une amende tout en laissant le quantum à sa discrétion.

Nous n'avons assisté qu'à quelques discussions où l'un ou l'autre des avocats, ou les deux, ont dû faire certains compromis explicites afin d'en arriver à un accord. Dans certains de ces dossiers, les procureurs de la Couronne et de la défense avaient au départ des points de vue différents, mais l'une des parties s'est finalement rangée à la suggestion de l'autre. En voici un exemple.

(3) (Entrave à un agent de la paix)

A.D.: C'est une entrave? Est-ce qu'il y a des choses particulières? Tu confirmes qu'il n'a pas d'antécédents?

P.C.: Oui.

A.D.: Qu'est-ce que tu demandes?

P.C.: C'est un cas d'amende.

A.D.: Cinquante dollars?

P.C.: Ben non! Cent dollars!

A.D.: O.K.

Dans quelques autres dossiers, il y eu compromis de part et d'autre, c'està-dire que chacun a mis de l'eau dans son vin afin d'en arriver à une entente. Comme l'exemple suivant permet de le constater, les concessions faites de part et d'autre sont habituellement peu considérables.

(4) (Possession pour fins de trafic de 0,5 gramme de haschisch, complot)

P.C.: C'est un trafic. (Il lit le précis de police.)

A.D.: Il a des antécédents?

P.C.: Oui, complot, deux vols avec violence, intro.

A.D.: Je pense qu'il travaille, je vais vérifier.

P.C.: Cinq cents dollars.

A.D.: Trois cents dollars.

P.C.: Quatre cents dollars.

A.D.: O.K.

Dans la majorité des négociations, il s'avère donc très facile pour les deux parties de s'entendre sur une suggestion commune de sentence. Les conflits, de même que les compromis de part et d'autre, sont extrêmement rares. Ce genre de négociations a déjà été observé aux États-Unis par Mather (1979) et Maynard (1984). À l'instar de ces derniers, nos données nous permettent d'affirmer que les compromis, lorsqu'il s'agit d'infractions mineures, ne constituent pas un élément nécessaire et essentiel de la négociation de 
plaidoyer et que les accords conclus relèvent davantage d'un processus routinier que de calculs rationnels.

\section{L'ÉCHEC DES NÉGOCIATIONS}

La négociation de plaidoyer a fréquemment été mise en opposition avec la tenue du procès. La contestation de l'accusation ne serait que le résultat de l'incapacité des avocats à régler le dossier par une négociation de plaidoyer ${ }^{7}$. Nos données, en ce qui concerne les négociations qui se rapportent à des délits mineurs, n'appuient pas cette assertion. Il semble exceptionnel que l'avocat de la défense choisisse de faire le procès lorsqu'il y a échec des négociations. Nous avons en effet constaté que devant l'impossibilité d'en arriver à un accord avec son confrère, l'avocat de la défense fait face à deux éventualités: 1) faire enregistrer un plaidoyer de culpabilité par son client et, le cas échéant, faire ses représentations sentencielles au juge; 2) remettre la cause et tenter de s'entendre ultérieurement avec un autre procureur. Les avocats de la défense rencontrés par Poirier (1986) confirment l'utilisation de l'une ou l'autre de ces stratégies lorsqu'ils sont confrontés avec cette situation.

Lorsque les négociations se trouvent dans une impasse, fréquemment, le procureur de la Couronne proposera à son collègue l'un des trois scénarios suivants:

1) que chacun fasse ses représentations sentencielles au juge, ce qui sousentend qu'il ne protestera pas avec vigueur à la suggestion de sentence de son confrère;

2) que les deux parties laissent la sentence à la discrétion du juge;

3) que le juge soit consulté hors cour: ce dernier indique la sentence qu'il imposera si l'accusé accepte de reconnaître sa culpabilité.

Habituellement, l'avocat de la défense accepte l'une ou l'autre des suggestions qui lui sont faites par la poursuite et règle le dossier par un plaidoyer de culpabilité. Lorsque malgré tout il y a remise de la cause, l'avocat de la défense a alors la possibilité de négocier avec un procureur de la Couronne différent. En effet, à l'exception des causes particulièrement graves comme celles de meurtre et de causes spécifiques comme celles d'agression sexuelle, les procureurs de la Couronne ne suivent pas la cause du début jusqu'à la fin des procédures. La plupart des procureurs sont plutôt assignés à une salle d'audience et sont saisis du dossier à une étape particulière du processus.

7. S'appuyant sur cette assertion, il a été largement affirmé que dans l'éventualité de l'abolition de la négociation de plaidoyer, on assisterait à une augmentation substantielle du nombre de procès. 
Ainsi, à l'exception d'un cas où il y a eu procès, dans tous les autres cas de remises, l'avocat de la défense a finalement incité son client à plaider coupable, selon toute vraisemblance après s'être entendu avec le nouveau procureur de la Couronne assigné au dossier. Nos données nous permettent donc d'affirmer que, contrairement à ce que plusieurs auteurs ont avancé, l'échec des négociations ne se traduit pas, lorsqu'il s'agit de délits mineurs, par la tenue du procès.

\section{LES OBJETS DE LA NÉGOCIATION}

\subsection{LES ACCUSATIONS}

Dans les négociations que nous avons observées, une réduction qualitative des accusations, c'est-à-dire un plaidoyer de culpabilité à une infraction de gravité moindre, ne s'est produite que dans trois dossiers. Par contre, la réduction quantitative des accusations (les retraits d'accusation) s'avère une pratique beaucoup plus répandue.

Ainsi, dans près de 7 dossiers sur 10 comportant plus d'un chef d'accusation, il y a eu retrait d'un ou de plusieurs d'entre eux. La majorité des retraits d'accusation ont eu lieu dans les dossiers où se retrouve le couple d'accusations « $\mathrm{vol}^{8} /$ possession illégale de biens volés». Dans 23 dossiers sur 29 , l'accusation de possession illégale de biens volés a été retirée et l'accusé a plaidé coupable à l'accusation de vol.

Ces retraits d'accusation se produisent lorsque les chefs d'accusation se recoupent ou, en d'autres mots, lorsque l'un des chefs d'accusation se trouve inclus dans l'autre ${ }^{9}$ : un des chefs doit alors être retiré parce que l'accusé ne peut, légalement, être reconnu coupable des deux chefs simultanément, en raison de la règle de droit qui établit qu'un individu ne peut être puni qu'une seule fois pour une même action prohibée.

La poursuite porte plus d'une accusation afin de s'assurer que l'accusé sera reconnu coupable d'au moins un chef d'accusation et ne sera pas acquitté ou libéré faute de preuves. Les procureurs de la Couronne sont d'ailleurs incités, lorsqu'ils ont en main une preuve suffisante pour amener une condamnation, à porter toutes les accusations qu'ils jugent nécessaires afin d'éviter

8. L'accusation de «vol» comprend aussi l'accusation d'introduction par effraction en vue de vol.

9. Par exemple, la possession d'un objet volé est incluse dans le vol: quand un individu vole un bien, il est nécessairement en possession d'un objet volé. Toutefois, il peut être en possession d'un objet volé sans être l'auteur du vol. 
que l'accusé ne ressorte indernne des procédures judiciaires entreprises contre lui (Procureur général du Québec, 1988).

Ces retraits d'accusation ne font qu'exceptionnellement l'objet de discussions préalables entre les deux parties. En effet, le plus souvent, l'avocat de la défense ne mentionne pas au procureur de la Couronne l'accusation à laquelle son client entend plaider coupable. Sans que les deux parties se le disent explicitement, chacun sait quel chef sera retiré. Ainsi, en cour, devant le juge, l'avocat de la défense est habituellement celui qui prend la parole le premier pour mentionner que son client désire enregistrer un plaidoyer de culpabilité à tel chef d'accusation, et le procureur de la Couronne poursuit en disant qu'il y a retrait de l'autre chef.

Les données recueillies montrent donc que la réduction de la gravité des accusations constitue une pratique inhabituelle de la poursuite mais que le retrait de certaines accusations est par ailleurs assez fréquent. Bien que l'on observe que la poursuite porte souvent deux accusations là où une seule suffirait, on note toutefois que cela ne lui procure pas pour autant une position privilégiée lors des négociations. En effet, le retrait de certaines accusations n'est que très rarement abordé lors des discussions entre les deux parties et s'effectue d'une manière plutôt routinière. On peut difficilement considérer ces retraits d'accusation comme des enjeux importants de la négociation et comme des avantages concédés à l'accusé: vraisemblablement, ce dernier aurait également bénéficié de ces retraits d'accusation lors du procès.

\subsection{LA SENTENCE}

La sentence constitue l'enjeu principal des négociations. En négociant, les avocats cherchent à s'entendre sur une suggestion commune de sentence à proposer au juge. Nos observations montrent que les deux parties ont souvent, à peu de choses près, la même perception du dossier, ce qui leur permet d'en arriver rapidement à un règlement qui les satisfait. Plusieurs auteurs (Maynard, 1984; Solomon, 1983; Feeley, 1979; Mather, 1979; Heumann, 1978) ont aussi constaté que les accords conclus lors d'une négociation de plaidoyer résultent d'une estimation réaliste de ce que «vaut» un cas.

En fait, au fil de leurs expériences, pour chaque catégorie d'infractions, les avocats établissent les caractéristiques du délit et du délinquant types (Sudnow, 1965). Lorsque le modus operandi et le profil psycho-social de l'accusé ne diffèrent pas ou peu de ceux qui se rapportent au délit et au délinquant types, l'évaluation de la peine méritée par l'accusé s'en trouve facilitée.

L'absence de divergences d'opinions entre les deux avocats sur la façon de régler le dossier peut donc s'expliquer par l'existence, d'ailleurs confirmée 
par d'autres études (entre autres: Nardulli et al., 1985; Feeley, 1979), de tarifs ou de barèmes sentenciels informels. Ces barèmes s'établissent à partir de la pratique quotidienne de chacun des intervenants. Ainsi, plus un délit est fréquemment jugé dans les cours d'un palais de justice, plus le tarif qui lui est rattaché est précis. À l'opposé, si on ne juge que quelques causes par année d'un délit particulier, le tarif sera plus difficile à identifier pour l'ensemble des intervenants.

Nos données tendent à démontrer une certaine homogénéité dans les sentences imposées à la suite d'une négociation de plaidoyer. Cette constance dans les décisions n'est pas une conséquence directe de la pratique de la négociation de plaidoyer, mais plutôt le résultat du fonctionnement des salles «à volume» dans lesquelles sont jugées journellement un grand nombre de causes du même type, ce qui favorise l'établissement et la connaissance des tarifs sentenciels informels. Cela explique en grande partie la rapidité avec laquelle les procureurs de la Couronne et de la défense parviennent à se mettre d'accord sur la sentence à proposer au juge.

Peut-on affirmer que l'accusé qui plaide coupable à une infraction mineure, suite à un accord conclu entre son avocat et le procureur de la Couronne, bénéficie ipso facto d'une réduction de sentence? Évidemment, la réponse à cette question demeure hypothétique puisqu'on ne connaît pas la sentence que le juge lui aurait imposée s'il avait été reconnu coupable à la suite d'un procès ou s'il avait plaidé coupable sans entente préalable avec le procureur de la Couronne. Certes, on ne peut nier que dans les salles d'audience à volume, et en particulier à la chambre de pratique, les procureurs de la Couronne adoptent lors des discussions une attitude conciliante qui favorise les ententes. Par contre, plusieurs éléments tendent à suggérer que les réductions de sentence, si réduction il y a, s'avèrent dans la plupart des cas minimes.

Il y a d'abord l'existence de tarifs sentenciels informels qui, comme nous venons de le montrer, minimisent les conflits au sujet de la recommandation de sentence à faire au juge. Deuxièmement, le fait qu'il s'agisse de délits mineurs diminue la marge de mancuvre dont dispose le procureur de la Couronne pour réduire la peine. En effet, ce dernier n'a pas le loisir de diminuer la sentence de quelques années d'emprisonnement, comme ce peut être le cas dans un dossier d'homicide, par exemple. Tout au plus l'accusé peut-il voir réduite sa période de détention de quelques mois ou économiser quelques dizaines ou centaines de dollars lorsqu'il s'agit d'une peine pécuniaire ${ }^{10}$.

10. En ce qui concerne les négociations observées, dans près de six cas sur dix les accusés ont reçu des sentences non carcérales: libération inconditionnelle, sentence suspendue, amende. Parmi les peines non carcérales, c'est surtout l'amende qui a été privilégiée. Quand 
Troisièmement, l'avocat de la défense ne possède pas, de manière générale, les éléments lui permettant d'exercer une certaine pression sur le procureur de la Couronne. En effet, selon les avocats de la défense rencontrés, dans la plupart des dossiers qui concernent des accusations mineures, la preuve retenue contre l'accusé est si forte que la culpabilité de ce dernier et donc sa condamnation à l'issue d'un procès ne font aucun doute dans l'esprit des deux parties. Il va sans dire que cette situation, si elle se vérifie ${ }^{11}$, limite les concessions que serait prêt à faire le procureur de la Couronne pour s'assurer un plaidoyer de culpabilité.

Nos données remettent donc en cause deux idées souvent véhiculées à propos de la négociation de plaidoyer soit: 1) qu'elle permet à l'accusé de bénéficier d'une réduction substantielle des accusations et de la sentence; 2) qu'elle produit des inégalités sentencielles entre les accusés.

\section{LES NÉGOCIATIONS DANS LES AFFAIRES D'HOMICIDE}

Parmi les dossiers d'homicide qui ont été étudiés, six comportaient une accusation de meurtre au premier degré, alors qu'une accusation de meurtre au second degré avait été portée dans les neuf autres dossiers. Avant d'aller plus loin, il convient, pour la compréhension de ce qui suit, de rappeler brièvement la nature des accusations et des peines relatives à l'homicide.

\section{NATURE DES ACCUSATIONS ET DES PEINES}

Dans les affaires d'homicide, plusieurs accusations sont possibles. Les plus fréquentes sont celles de meurtre au premier degré, meurtre au second degré, homicide involontaire coupable et négligence criminelle causant la mort. Le meurtre prémédité ${ }^{12}$ constitue un meurtre au premier degré. Le meurtre non prémédité, qui n'est pas un homicide involontaire coupable, constitue un meurtre au second degré. La différence entre le meurtre, qu'il soit

l'incarcération a été imposée, il s'agit dans la moitié des cas de peines de moins de trois mois de détention.

11. Aux États-Unis, Mather (1979) et Heumann (1978) ont recueilli des propos similaires de la part des avocats de la défense: la majorité d'entre eux estimaient que, dans la plupart de leurs dossiers, la validité de la preuve présentée par la poursuite ne pouvait être contestée. Toutefois, certains mettent en doute ce qu'ils considèrent comme une justification a posteriori de la décision de négocier un plaidoyer de culpabilité. Ainsi, un des avocats de la défense rencontrés affirme que si davantage de procès étaient tenus, plusieurs d'entre eux, quoi que ses collègues en pensent, se termineraient par l'acquittement de l'accusé.

12. L'homicide d'un agent de la paix et l'homicide commis au cours d'un détournement d'avion, d'une agression sexuelle, d'un enlèvement ou d'une séquestration constitue également un meurtre au premier degré. 
au premier ou au second degré, et l'homicide involontaire coupable se trouve dans l'intention de tuer. Lorsqu'il porte une accusation de meurtre, le ministère public doit prouver que l'accusé avait l'intention spécifique de tuer la victime. Ce n'est pas le cas pour l'homicide involontaire. Le législateur considère, en effet, qu'il y a homicide involontaire lorsque l'accusé n'avait pas l'intention de tuer la victime au moment où il a commis les gestes qui se sont avérés fatals. Ajoutons que très souvent les tribunaux jugent qu'il y a homicide involontaire lorsque l'accusé a agi sous le coup de la colère ou après provocation.

Quant à l'infraction de négligence criminelle, le législateur la définit de la façon suivante:

Est coupable de négligence criminelle quiconque,

a) en faisant quelque chose, ou

b) en omettant de faire quelque chose qu'il est de son devoir d'accomplir,

montre une insouciance déréglée ou téméraire à l'égard de la vie ou de la sécurité d'autrui.

(Art. 219 du Code criminel.)

Pour les infractions de meurtre, la peine minimale obligatoire prévue au Code criminel est l'emprisonnement à perpétuité. L'accusé est admissible à une libération conditionnelle après au moins 25 ans de détention lorsqu'il s'agit d'un meurtre au premier degré, et au moins 10 ans lorsqu'il s'agit d'un meurtre au second degré. Toutefois, dans ce dernier cas, le juge possède la liberté d'augmenter le délai préalable à la libération conditionnelle à au plus 25 ans, si des circonstances exceptionnelles le justifient. Quant aux infractions d'homicide involontaire et de négligence criminelle, elles sont également passibles de l'emprisonnement à perpétuité, mais il s'agit d'une peine maximale : aucune peine minimale n'est prévue pour ces deux infractions.

\section{LE DÉROULEMENT DES NÉGOCIATIONS}

Dans les dossiers d'homicide, les discussions au sujet d'un éventuel plaidoyer de culpabilité de la part de l'accusé s'étendent sur plusieurs jours, voire plusieurs semaines. Propositions et contre-propositions se succèdent avant d'en arriver à un accord entre les deux parties.

Encore une fois, accusés et, dans le cas présent, familles des victimes se trouvent le plus souvent exclus des discussions. Toutefois, les policiers y jouent un rôle plus actif que dans les négociations qui concernent les délits mineurs, comme nous le verrons plus loin. 
Les négociations se déroulent selon un procédé transactionnel : l'accusé n'acceptera de reconnaître sa culpabilité que s'il reçoit, en retour, certaines concessions de la part de la poursuite. C'est pourquoi les négociations sont avant tout gouvernées par le rapport de forces existant entre les deux parties. Nous verrons un peu plus loin que plusieurs facteurs, en particulier la validité de la preuve retenue contre l'accusé, contribuent à faire pencher le rapport đe forces d'un côté ou de l'autre et influencent les modalités de l'entente qui sera conclue.

En tout temps, la menace de la tenue du procès, dans l'éventualité d'un désaccord, plane au-dessus des discussions. La contestation de l'accusation constitue la conséquence immédiate de l'échec des négociations. Or, les procès pour meurtre exigent des avocats un travail de préparation considérable et peuvent se prolonger pendant plusieurs semaines, selon la preuve et la contrepreuve qui y sont présentées. En outre, ils se tiennent devant jury, et les avocats rencontrés s'entendent pour affirmer que les jurés rendent des décisions tellement imprévisibles qu'ils préfèrent le plus souvent négocier plutôt que de s'engager dans une contestation de l'accusation. L'imminence du procès oblige les deux parties à mettre un terme à leurs tergiversations: c'est fréquemment quelques jours avant la tenue de celui-ci, parfois le matin même, que les deux parties finissent par trouver un terrain d'entente.

Il nous faut insister sur un aspect particulier des dossiers d'homicide qui a une incidence considérable sur la façon dont ils se règlent: il s'agit de la peine minimale obligatoire d'emprisonnement à perpétuité que se voit infliger l'accusé reconnu coupable de meurtre au premier ou au second degré. Dès le départ, cette situation déséquilibre le rapport de forces en faveur de la poursuite. Car elle pose un dilemme sérieux à l'accusé et à son avocat: risquer le procès, lequel peut se terminer à un extrême par un acquittement et à l'autre extrême par une condamnation à l'accusation originale et l'emprisonnement à perpétuité, ou plaider coupable à une infraction moindre et se voir imposer une sentence moindre. Devant une telle alternative, certains individus sont tentés, malgré les possibilités réelles d'acquittement, de renoncer à un procès qui pourrait se terminer par une condamnation.

Voici deux exemples contrastés de négociations qui se sont produites dans les dossiers étudiés. 


\section{Dossier 1}

L'accusé fait face à une accusation de meurtre au premier degré. Dès le début des procédures judiciaires, l'avocat de la défense propose au procureur de la Couronne de régler le dossier par un plaidoyer de culpabilité à une infraction moindre de négligence criminelle entraînant la mort. Le procureur de la Couronne, sans fermer la porte totalement à la négociation, préfère attendre la tenue de l'enquête préliminaire afin d'obtenir davantage d'informations concernant les faits de la cause. Au terme de l'enquête préliminaire, le juge estime que la preuve présentée devant lui n'est pas suffisante pour appuyer l'accusation originale de meurtre au premier degré. Une accusation de meurtre au second degré est alors portée contre l'accusé.

À l'offre de l'avocat de la défense de régler pour une accusation de négligence criminelle entraînant la mort, le procureur de la Couronne répond en proposant une réduction à homicide involontaire. L'avocat de la défense présente la proposition à son client, tout en lui recommandant de ne pas l'accepter. Ce demier refuse la proposition et le procureur de la Couronne finit par se rallier à l'offre de son collègue: il accepte de réduire l'accusation de meurtre au second degré à celle de négligence criminelle entraînant la mort. L'avocat de la défense propose alors de s'entendre sur une suggestion commune de sentence d'un an d'incarcération. Le procureur de la Couronne préfere 18 mois. L'avocat de la défense se range finalement à l'avis de son collègue.

\section{Dossier 2}

Depuis le début des procédures, le procureur de la Couronne refuse de négocier et rejette la proposition de la défense de réduire l'accusation de meurtre au premier degré à celle de meurtre au second degré. Devant l'insistance de l'avocat de la défense, le procureur de la Couronne lui fait la proposition suivante: plaidoyer de culpabilité à l'accusation de meurtre au second degré et suggestion commune d'une période de détention de 20 ans avant d'être admissible à une libération conditionnelle. L'avocat de la défense réplique que la concession est minime et fait une contre-offre: 18 ans. Le procureur de la Couronne refuse et l'avocat de la défense n'a pas d'autre choix que d'accepter la proposition de ce dernier, parce qu'il considère que les preuves retenues contre son client pourraient lui valoir une condamnation pour meurtre au premier degré. 


\section{LES ENTENTES CONCLUES}

\subsection{LA RÉDUCTION DE L'ACCUSATION}

La réduction qualitative de l'accusation constitue le point essentiel des discussions, bien avant la sentence. En raison des peines minimales obligatoires, les ententes doivent nécessairement comprendre une diminution de la gravité de l'accusation pour que l'accusé puisse bénéficier d'une éventuelle réduction de sentence.

Dans quatre des 15 dossiers étudiés, il y a eu réduction de l'accusation originale de meurtre au premier degré à celle de meurtre au second degré. Chaque fois, l'accusé n'a pas bénéficié de la règle qui veut que le juge fixe à 10 ans le moment d'admissibilité à une libération conditionnelle. Dans deux de ces dossiers, les parties ont recommandé que l'accusé ne puisse bénéficier d'une libération conditionnelle avant 20 ans, suggestion que le juge a entérinée. L'accusé a donc profité, théoriquement, d'une réduction de sentence de cing ans. Dans le troisième dossier, l'accusé s'est vu imposer 18 ans de détention fermes avant d'être admissible à une libération conditionnelle. Enfin, dans le dernier dossier, les deux avocats ont suggéré conjointement que l'accusé ne puisse bénéficier d'une libération conditionnelle avant 10 ans, mais le juge a refusé de suivre cette recommandation et a augmenté la période d'admissibilité à 25 ans. Cela signifie que la réduction de l'accusation consentie par la poursuite n'a eu, dans ce dossier, aucun impact sur la sentence: en dépit de son plaidoyer de culpabilité à l'accusation de meurtre au second degré, l'accusé a reçu la peine prévue pour le meurtre au premier degré.

Comme on peut le constater, la réduction de l'accusation de meurtre au premier degré à celle de meurtre au second degré n'a que peu de répercussions ou, tout au plus, un effet incertain sur la sentence. L'avantage que retire l'accusé, dans la plupart des cas mais pas tous, c'est d'être admissible plus rapidement à une libération conditionnelle.

C'est lorsque la poursuite accepte de réduire l'accusation originale de meurtre au premier ou au second degré à celle d'homicide involontaire ou de négligence criminelle causant la mort que les avantages que retire l'accusé sur le plan de la sentence sont les plus considérables. Dans les dossiers où la poursuite a consenti à une telle réduction de l'accusation, les sentences que l'on a imposées aux accusés s'étendent d'un sursis de sentence, accompagné d'une ordonnance de travaux communautaires, à l'emprisonnement pour une période de 22 ans.

À première vue, il semble évident que la réduction des accusations, surtout lorsque les accusations originales de meurtre au premier ou au second 
degré sont remplacées par celles d'homicide involontaire ou de négligence criminelle entraînant la mort, donne à l'accusé l'occasion de bénéficier d'une réduction de sentence ${ }^{13}$. Mais cette assertion n'est vraie que dans la mesure où l'accusation originale correspond au délit perpétré. Si le procureur de la Couronne porte, dès le départ, une accusation plus grave que la preuve en sa possession ne lui permet de le faire, la réduction de l'accusation ne constitue qu'un bénéfice illusoire pour l'accusé ${ }^{14}$.

Les données recueillies dans le cadre de cette recherche ne nous permettent pas de statuer sur la justesse des accusations portées par le procureur de la Couronne dans les dossiers de meurtre. Ce que nous avons pu constater, néanmoins, c'est que fréquemment les procureurs de la Couronne et de la défense estimaient qu'il existait une probabilité réelle que l'accusé soit reconnu coupable de l'accusation originale mais qu'il y avait également de fortes possibilités qu'il soit reconnu coupable d'une infraction moindre et même, dans certains cas, qu'il soit acquitté. Comme nous l'avons déjà mentionné, les avocats rencontrés estiment que les décisions des jurés dans les procès pour meurtre manquent de constance et de prévisibilité. Devant l'issue incertaine du procès, les deux parties, mais surtout l'accusé, préfèrent souvent l'assurance d'un résultat mitoyen prenant la forme d'un plaidoyer de culpabilité à une accusation réduite.

\subsection{LES SUGGESTIONS COMMUNES DE SENTENCE}

La plupart du temps, la réduction de l'accusation est accompagnée d'une suggestion commune de sentence. Dans certains cas, la poursuite consent à réduire l'accusation, à la condition sine qua non que l'avocat de la dëfense accepte de recommander la sentence qu'elle a au préalable déterminée. Si ce dernier refuse la proposition, le procureur de la Couronne le menace de recourir au procès. Placé devant cet ultimatum, l'avocat de la défense, ne voulant pas prendre le risque du procès, peut accepter le marché même s’il estime que la sentence proposée est beaucoup trop sévère.

Toutefois, les ententes conclues ne comportent pas toujours une telle condition. Ainsi, dans quatre des dossiers étudiês, les avocats se sont abstenus de faire une recommandation commune de sentence au juge. Dans un des dossiers, les deux parties n'arrivaient pas à se mettre d'accord sur un quantum

13. Encore une fois, parce que l'on ne peut présumer de l'accusation pour laquelle l'accusé aurait été trouvé coupable et de la sentence imposée, il est impossible d'évaluer si l'accusé, en bénéficiant d'une réduction de l'accusation, profite du mêrne coup d'une diminution de sentence.

14. Les écrits portant sur la négociation de plaidoyer font grand état d'une pratique qui inciterait fortement les accusés à plaider coupable: l'overcharging, soit l'aggravation et la multiplication artificielle des accusations portées contre un individu. 
de sentence satisfaisant pour tous. Elles se sont finalement entendues sur la modalité suivante: le procureur de la Couronne s'est engagé à ne pas demander plus de 20 ans de détention fermes avant que l'accusé ne soit admissible à une libération conditionnelle, tandis que l'avocat de la défense a accepté de ne pas suggérer moins de 15 ans fermes.

Dans les trois autres dossiers, le procureur de la Couronne a refusé que les deux parties présentent une suggestion commune au juge, tout en assurant à son collègue qu'il n'exigerait pas une sentence extrêmement sévère lors des représentations sentencielles. En fait, dans les trois dossiers, il y a eu demande qu'un rapport présentenciel soit effectué. L'entente plus ou moins explicite conclue entre les deux parties était la suivante: si le rapport présentenciel était favorable à l'accusé, la poursuite s'engageait à ne pas s'opposer aux recommandations du rapport. Un procureur de la Couronne qui a conclu ce type d'entente a justifié sa position en expliquant qu'il n'avait pas voulu prendre la responsabilité de recommander une sentence clémente pour un crime aussi grave que l'homicide involontaire.

Comme on peut le constater, pour une même infraction, les modalités de l'accord peuvent varier d'un dossier à l'autre. En fait, plusieurs facteurs contribuent à faire pencher le rapport de forces d'un côté ou de l'autre et sont susceptibles d'influencer l'étendue des concessions que la poursuite sera prête à faire, y compris, dans certains cas, le refus de toute négociation. Certains de ces facteurs peuvent également intervenir lors des discussions au sujet des délits mineurs, mais leur influence demeure beaucoup plus importante dans les affaires d'homicide. Nous en discutons dans la prochaine partie.

\section{LES FACTEURS QUI INFLUENCENT LE RÉSULTAT DES NÉGOCIATIONS}

\subsection{LA VALIDITÉ DE LA PREUVE}

La force de la preuve constitue, sans nul doute, l'élément le plus déterminant de tous. Une preuve forte place la poursuite dans une position privilégiée pour négocier: elle peut en tout temps invoquer la menace du procès et les conséquences dramatiques que cela pourrait signifier pour l'accusé afin de contraindre l'avocat de la défense à accepter l'offre qu'elle lui propose. Dans de tels cas, ce dernier n'a que rarement d'autre choix que d'accepter la proposition de la poursuite, mêrne s'il la trouve inappropriée, puisque le procès ne peut que se terminer par une condamnation et, vraisemblablement, par l'emprisonnement à perpétuité de l'accusé ${ }^{15}$.

15. Dans les exemples de négociations que nous avons présentés, la poursuite possédait 
Lorsque les preuves retenues contre l'accusé sont plus faibles, le procureur de la Couronne perd sa position de force et est obligé de faire preuve d'une certaine souplesse s'il ne veut pas courir le risque du procès. Certains (Poirier, 1986) ont déjà souligné que lorsque la preuve de la poursuite comporte certaines faiblesses, l'avocat de la défense se retrouve dans une situation très inconfortable: il doit décider de négocier ou non alors que la probabilité d'un acquittement lors d'un procès, quoique faible dans certains cas, demeure bien réelle. Encore une fois, c'est la certitude du résultat qui sera le facteur prépondérant: des deux côtés, on préfère la certitude au risque que constitue la tenue du procès. Et c'est souvent l'accusé qui exige cette certitude. Par exemple, dans un des dossiers étudiés, l'avocat de la défense considérait qu'il $\mathrm{y}$ avait de fortes probabilités que son client soit acquitté. Néanmoins, ce dernier n'a pas voulu assumer le risque du procès et a accepté l'offre de la poursuite qui lui proposait une sentence extrêmement clémente.

\subsection{LES CIRCONSTANCES AGGRAVANTES ET ATTÉNUANTES}

Le Code criminel stipule que tous les individus reconnus coupables de meurtre doivent purger la même peine, soit l'emprisonnement à perpétuité. Si l'on fait abstraction de la possibilité d'être admissible à une libération conditionnelle plus rapidement pour certains d'entre eux, cela signifie donc que dans l'esprit du législateur, tous les meurtriers doivent être traités de la même façon. Toutefois, dans la pratique quotidienne des tribunaux, certains meurtres sont jugés subjectivement moins graves que d'autres, selon les circonstances qui ont mené au crime et les protagonistes en cause. Les procureurs de la Couronne et de la défense nous ont dit comparer la gravité des affaires d'homicide entre elles: selon leurs propres termes, «il y a des affaires plus sympathiques que d'autres». Ainsi, certains meurtres, de l'avis de tous, méritent clémence. Prenons par exemple le cas d'une mère souffrant d'une grave dépression qui, de manière préméditée, met fin aux jours de son enfant. Il est indéniable qu'il s'agit, par sa nature, d'un acte différent de l'homicide commis par un tueur à gages. Toutefois, la loi prévoit, dans un cas comme dans l'autre, l'emprisonnement à perpétuité.

Le mobile est très important pour évaluer la gravité subjective du délit. Lorsque le suspect a agi sans mobile apparent ou lorsque le mobile est le vol, le crime est généralement qualifié de sordide. Dans la plupart de ces cas, l'agresseur ne connaissait pas ou très peu sa victime. Les négociations dans de tels dossiers sont habituellement très difficiles: le procureur de la Couronne est peu disposé à faire des compromis à moins que les risques d'acquittement

de très fortes preuves contre l'accusé dans le dossier 2 , alors que cette preuve comportait des faiblesses certaines dans le dossier 1 . 
ou de condamnation à une accusation moindre ne soient élevés. Quand la poursuite consent à négocier, les avantages que retire l'accusế sont le plus souvent très minimes. La plupart des dossiers étudiés qui se sont terminés par un plaidoyer de culpabilité à l'accusation de meurtre au second degré font partie de cette catégorie.

À l'inverse, s'il est possible de trouver une justification au geste commis par l'accusé (il a été provoqué par la victime, il a agi sous le coup de la peur ou de la colère, il souffrait de problèmes psychologiques) et si, en outre, il s'agit d'un individu qui n'est pas criminalisé, qui regrette son geste et qui a collaboré avec la justice, la poursuite consentira plus facilement à une diminution des accusations, le plus souvent une réduction à l'accusation d'homicide involontaire ou de négligence criminelle, et par ricochet à une diminution de la sentence. De manière générale, dans de tels cas, l'accusé et la victime se connaissaient et entretenaient une relation amicale ou amoureuse.

\subsection{LES POLICIERS}

Le rôle des policiers dans la négociation ne doit pas être sous-estimé. $\mathrm{Si}$, somme toute, ils sont plutôt absents des négociations qui se déroulent dans les dossiers mineurs, nous avons constaté que dans celles qui ont lieu dans les dossiers de meurtre, ils jouent fréquemment un rôle de premier plan. Lorsqu'il s'agit d'une affaire d'homicide, parce qu'ils font partie d'une escouade spécialisée, qu'ils doivent mener des enquêtes qui exigent un travail considérable et qu'ils sont appelés à collaborer très étroitement avec les procureurs de la Couronne, les policiers se sentent davantage concernés par la conclusion de la cause.

Leur influence s'exerce de deux manières. D'abord, ils peuvent influencer indirectement le procureur de la Couronne en lui communiquant leur perception du dossier et du suspect. Ensuite, les policiers peuvent faire pression directement sur le procureur de la Couronne en s'opposant à toute modification de l'accusation originale (bloquant pour ainsi dire toute possibilité de négociation) ou à une suggestion de sentence qu'ils estiment inappropriée.

Bien que certains procureurs de la Couronne, surtout parmi les plus expérimentés, démontrent une grande indépendance d'esprit envers l'opinion des policiers, nul doute que le point de vue de ces derniers est pris en considération dans la plupart des dossiers.

\subsection{LE JUGE}

Règle générale, les juges ne participent pas directement aux discussions qui ont pour but de déterminer les modalités d'une entente. Ils peuvent cepen- 
dant être consultés préalablement à l'audition de la cause. Lors de ces rencontres informelles, le juge est mis au courant des circonstances de l'affaire et de l'entente conclue entre les deux avocats. Le but de l'entreprise est de s'assurer que le juge acceptera la suggestion commune à laquelle les deux parties en sont arrivées, puisqu'il n'est pas légalement lié par les ententes: il peut entériner ou non les recommandations qui lui sont faites.

Toutefois, ce ne sont pas tous les juges qui acceptent d'être consultés: la plupart des juges de la Cour supérieure refusent de rencontrer les avocats hors cour, alors que ceux de la Cour du Québec y consentent volontiers ${ }^{16}$. Parce qu'ils refusent d'indiquer aux deux parties, préalablement à l'enregistrement du plaidoyer de culpabilité, s'ils considèrent l'entente acceptable ou non, nos données suggèrent que le risque que l'entente ne soit pas entérinée par le juge augmente lorsqu'il s'agit d'un juge de la Cour supérieure. Il va sans dire que les conséquences d'une telle situation peuvent s'avérer désastreuses pour l'accusé. Un suspect, comme c'est arrivé dans un des dossiers étudiés, peut se voir infliger une peine de 25 ans de détention avant d'être admissible à une libération conditionnelle, alors qu'il avait reconnu sa culpabilité en croyant écoper de seulement 10 ans d'emprisonnement fermes, comme les deux avocats en avaient convenu et l'avaient suggéré au juge.

Les juges de la Cour supérieure influencent donc indirectement les modalitês des ententes conclues: les avocats tiendront compte de la philosophie pénale et du niveau de sévérité du juge assigné à la cause avant de se mettre d'accord sur une recommandation commune. La situation est totalement différente lorsque le procureur de la Couronne accepte de réduire l'accusation originale à celle d'homicide involontaire ou de négligence criminelle. Il dépose alors un nouvel acte d'accusation et la cause change de juridiction : elle passe de la Cour supérieure à la Cour du Québec. Lorsque cela se produit, les deux parties peuvent dans une grande mesure choisir le juge qui recevra le plaidoyer de culpabilité. Les avocats auront pris soin de vérifier, au préalable, si ce demier estime acceptable la proposition commune qu'ils lui suggèrent. Dans la négative, les avocats auront la possibilité de sélectionner un autre juge qui sera plus réceptif à leur proposition.

\subsection{LES RELATIONS INTERPERSONNELLES}

Plusieurs avocats, tant de la défense que de la poursuite, soutiennent que les relations cordiales qu'ils entretiennent avec certains de leurs collègues

16. Rappelons que les juges de la Cour supérieure président, avec un jury, les procès pour lesquels un suspect doit répondre à une accusation de meurtre au premier ou au second degré. Lorsqu'il s'agit d'une accusation d'homicide involontaire ou de négligence criminelle, la cause peut être entendue par un juge de la Cour du Québec. 
peuvent favoriser les négociations ou, au contraire, les rendre plus difficiles. Ainsi, certains procureurs de la Couronne, afin de ne pas être accusés de favoritisme, adopteraient une attitude plus rigide lorsqu'ils négocient avec des amis. Par ailleurs, l'animosité entre deux avocats pourrait, dans plusieurs cas, faire échouer toute tentative de négociation.

\subsection{LA COMPÉTENCE PROFESSIONNELLE DES AVOCATS}

L'expérience et l'habileté professionnelle des avocats qui se font face lors d'une négociation peuvent sans aucun doute influer sur le résultat de celle-ci. Devant un adversaire professionnellement aguerri, possédant très bien l'art de l'interrogatoire, du contre-interrogatoire et de la plaidoirie, qui s'est établi avec les années une réputation de gagnant, la perspective d'un procès risque de faire peur. L'autre partie peut alors se montrer pius conciliante et être prête à faire de plus grandes concessions afin d'éviter un procès où elle pourrait perdre la face. Dans de tels cas, la menace du procès, implicite ou explicite, constitue un atout dans le jeu de l'avocat le plus combatif et le plus compétent.

\subsection{LA COUVERTURE MÉDIATIQUE DU DOSSIER}

L'intérêt que les médias et le public portent à l'affaire peut inciter à la prudence un procureur de la Couronne qui préférera suivre la procédure officielle et faire le procès plutôt que de s'exposer à des critiques éventuelles en négociant. Dans un des dossiers étudiés, dans lequel un individu a tué sa conjointe, le procureur de la Couronne a admis que sa décision de refuser toute négociation pouvait s'expliquer en partie par ce motif.

Par ailleurs, si le procureur de la Couronne décide, malgré tout, de régler le dossier par la voie de la négociation, il fera à nouveau preuve de prudence en maintenant une attitude stricte face aux demandes de l'accusé et en réduisant les avantages dont il pourrait le faire bénéficier.

\section{DES IMPACTS DIFFÉRENTIELS}

Les données que nous venons de présenter montrent clairement que les enjeux et les conséquences de la négociation de plaidoyer sont sensiblement différents selon que l'accusé fait face à une accusation mineure ou à une accusation de meurtre.

Dans le premier cas, il s'agit de négociations réduites à leur plus simple expression. Les négociations s'apparentent à un processus routinier qui relève davantage de la coopération entre les parties que de tractations visant à obtenir des aveux de culpabilité en promettant au suspect des avantages de plusieurs 
ordres, prenant surtout la forme d'une réduction de sentence. Ainsi, la décision de plaider coupable est rarement conditionnelle à l'offre faite par la poursuite. Lorsqu'il se présente pour négocier, l'avocat de la défense sait déjà que son client accepte de reconnaître sa culpabilité. Le plaidoyer de culpabilité ne constitue pas un élément de la négociation puisque advenant l'échec de celle-ci, l'avocat de la défense ne s'engagera pas dans un procès mais tentera plutôt de régler le dossier avec un autre procureur. Les discussions ne visent donc qu'à s'entendre sur ce que «vaut» le cas, c'est-à-dire sur la peine qui est habituellement imposée pour une telle infraction.

La réalité est tout autre lorsqu'il s'agit d'une accusation de meurtre. Les négociations de plaidoyer qui se déroulent dans un tel contexte rejoignent la vision traditionnelle de la négociation de plaidoyer, dans laquelle le rapport de forces tient une place prépondérante. Dans les dossiers d'homicide, en contrepartie de ses aveux de culpabilité, la poursuite permet à l'accusé d'obtenir une certitude concernant la conclusion du dossier et, dans certains cas, de bénéficier d'une réduction de sentence.

Plusieurs auteurs ont affirmé, à l'encontre de la négociation de plaidoyer, qu'une telle pratique fait en sorte qu'il est possible qu'un suspect reconnaisse sa culpabilité à des gestes qu'il n'a pas commis par crainte d'être condamné lors d'un procès et de se voir infliger une sentence plus sévère. En ce qui concerne les dossiers d'homicide, il est préférable de délaisser la dichotomie innocent/coupable et de poser le problème en termes de culpabilité légale et factuelle. Lorsqu'une accusation de meurtre au premier ou au second degré est portée, souvent l'identification de l'accusé comme étant l'auteur des gestes qu'on lui reproche, donc sa culpabilité factuelle, ne fait aucun doute. Toutefois, sur le plan légal, cela ne fait pas de lui un coupable pour autant: plusieurs défenses peuvent être invoquées (provocation, légitime défense, intoxication, folie, etc.) afin de le disculper ou de nuancer sa culpabilité factuelle. Plutôt que de circonscrire la question aux individus innocents que la négociation de plaidoyer pourrait inciter à plaider coupable, il vaut donc mieux l'élargir en intégrant ceux qui, tout en ayant commis les gestes dont on les accuse, auraient pu être acquittés ou condamnés à une infraction moindre lors du procès parce que leur culpabilité légale au regard de l'accusation originale n'aurait pu être prouvée.

Quelle que soit l'accusation à laquelle doit répondre un accusé, qu'il s'agisse d'un délit mineur ou plus sérieux, cette possibilité que nous venons d'énoncer existe. Toutefois, le risque semble plus important dans les dossiers d'homicide, en raison de la peine minimale d'emprisonnement à perpétuité prévue par le législateur pour les accusations de meurtre au premier et au second degré. Ces peines minimales extrêmement sévères constituent pour 
l'accusé un incitatif puissant à reconnaître sa culpabilité à une infraction moindre, souvent un homicide involontaire, pour laquelle aucune peine minimale n'est prévue. Lorsque le procureur de la Couronne lui offre la possibilite de bénéficier d'une telle réduction de l'accusation, il peut s'avérer difficile pour un accusé de refuser la proposition. En ce sens, les peines minimales obligatoires constituent en soi des pressions indues exercées sur l'accusé afin d'obtenir des aveux de culpabilité. Cette situation oblige à relativiser les avantages obtenus par l'accusé en échange de son plaidoyer de culpabilité puisqu'il semble tout à fait plausible qu'un accusé, par peur d'être condamné à passer le reste de sa vie derrière les barreaux, consente à avouer sa culpabilité à une infraction moindre, alors qu'il aurait pu être acquitté ou trouvé coupable de cette accusation moindre lors du procès.

Un autre élément peut s'ajouter à la pression qu'exerce sur l'accusé une réduction de l'accusation originale: il s'agit des moyens financiers dont il dispose. En effet, les données que nous avons recueillies semblent démontrer que la qualité de la représentation d'un accusé dépend en grande partie de la somme d'argent qu'il peut débourser pour les honoraires de son avocat. Non seulement l'argent permet-il de se payer un meilleur avocat, mais il permet aussi de s'offrir un procès devant jury. Or, un tel procès coûte cher en honoraires, et il semble bien que très peu d'accusés, dans de telles circonstances, peuvent débourser l'argent requis pour leur défense. Un avocat de la défense, que nous avons rencontré pour l'un des dossiers, a admis qu'il n'avait jamais envisagé de contester l'accusation, son client n'étant pas en mesure de payer les frais que cela aurait exigés ${ }^{17}$.

Une autre difficulté soulevée par la pratique de la négociation de plaidoyer dans les affaires d'homicide concerne le traitement inégal des accusés: ce ne sont pas tous les accusés qui ont la possibilité de négocier leur plaidoyer, et parmi ceux qui le font, il peut exister, pour des cas semblables, des différences plus ou moins importantes entre les accords conclus.

En effet, bien qu'il semble s'agir d'une infime minorité, certains individus accusés de meurtre n'ont pas la possibilité de négocier leur plaidoyer, la poursuite refusant de s'engager dans une telle négociation. Outre la validité de la preuve et la présence de circonstances atténuantes ou aggravantes, qui constituent dans une certaine mesure des éléments objectifs, d'autres facteurs,

17. Il se peut que les individus accusés de meurtre qui sont représentés par un avocat employé permanent de l'Aide juridique ne subissent pas les mêmes pressions pour plaider coupable que ceux qui sont représentés par un avocat de pratique privée. Il serait intéressant de vérifier si les procès ne sont pas plus fréquents lorsque l'accusé est représenté par un avocat de l'Aide juridique. Ces derniers seraient peut-être davantage portés, lorsqu'une possibilité réelle d'acquittement existe, à tenir le procès. 
plus subjectifs, peuvent expliquer l'attitude du procureur de la Couronne, tels la couverture médiatique du dossier, les relations existant entre les deux avocats, l'habileté professionnelle de chacun, la perception du dossier par les policiers. Dans ]'éventualité où le procureur de la Couronne accepte de négocier, tous ces éléments auront également une influence sur l'entente qui sera conclue.

En somme, la négociation de plaidoyer dans les affaires d'homicide constitue selon toute vraisemblance une pratique qui, virtuellement, ouvre la porte aux inégalités. Cela ne signifie pas, toutefois, que le procès soit plus équitable. La conclusion de celui-ci est également influencée, entre autres, par l'habileté professionnelle et l'expérience des avocats ainsi que par la personnalité du juge et des jurés. En ce sens, il ne semble pas que l'on puisse affirmer que les sentences imposées à l'issue d'un procès soient plus rationnelles que celles imposées à la suite d'une négociation de plaidoyer. À cet égard, rappelons qu'au Canada, Hogarth (1971) a démontré que les juges sont loin de partager la même philosophie pénale et que chacun utilise des principes sentenciels différents dans la détermination de la peine à infliger à un accusé.

Les différences dans le traitement des accusés sont beaucoup moindres lorsqu'il s'agit d'accusations mineures. L'institution de la chambre de pratique, qui est considérée comme la salle des négociations, fait en sorte qu'il est toujours possible de négocier si l'avocat de la défense le désire. En outre, s'il ne réussit pas à s'entendre avec la poursuite, l'avocat de la défense peut toujours esayer de conclure, à une étape ultérieure, un accord satisfaisant avec un autre procureur. Par ailleurs, s'il peut exister, lorsqu'il s'agit d'affaires mineures, des différences dans les modalités des accords conclus pour des dossiers semblables, l'existence de tarifs sentenciels informels a pour effet de réduire ces disparités potentielles.

\section{CONCLUSION}

La négociation de plaidoyer telle que nous l'avons définie se pratique sur une grande échelle au palais de justice de Montréal. On peut même affirmer que c'est la façon la plus commune de régler les dossiers. Majs il ne s'agit pas d'une pratique uniforme. Les deux types de négociation que nous avons identifiés représentent vraisemblablement les deux extrêmes d'un spectre continu. Entre ces deux extrêmes se retrouvent des dossiers de gravité moyenne, pour lesquels les négociations s'apparentent davantage à l'une ou l'autre forme de négociation.

La fréquence de ces deux types de négociation n'est pas équivalente. Puisque les dossiers mineurs constituent la plus grande partie du contentieux 
des causes criminelles à Montréal, la forme de négociation que l'on rencontre dans ces dossiers est nécessairement celle que l'on retrouve le plus souvent.

Les négociations qui se déroulent dans les dossiers d'homicide soulèvent des difficultés majeures dont la possibilité que des suspects enregistrent un plaidoyer de culpabilité alors qu'ils auraient pu être acquittés ou reconnus coupables d'une infraction moindre lors du procès et le traitement inégal des accusés apparaissent les plus fondamentales. Ces problèmes, s'ils existent, sont moins préoccupants lorsqu'il s'agit de délits mineurs.

Les résultats obtenus mettent en évidence, croyons-nous, l'importance de bien connaître les enjeux et les conséquences de la négociation de plaidoyer avant de statuer sur la légitimité de la pratique et de procéder à une réforme visant le contrôle ou l'encadrement de celle-ci.

D'autres problèmes, qui n'ont pas été traités dans le cadre de cet article, sont associés à la négociation de plaidoyer. Citons celui de l'exclusion des victimes et des accusés de toutes les discussions entourant le règlement de la cause. À la suite de leur expérience, les deux groupes expriment souvent les mêmes sentiments: ils regrettent de n'avoir pas eu la possibilité de s'expliquer, de donner leur version des faits et ont l'impression de s'être fait flouer par le système. Les préjugés qui ont cours dans la population sont également bien ancrés chez les victimes et les accusés: ils estiment que la justice n'existe pas ou bien qu'elle bénéficie aux mieux-nantis.

Le fait que les décisions soient prises en secret, entre les deux avocats et quelquefois le juge, derrière des portes closes, discrédite la justice. Cette gestion de la justice en catimini projette dans le public une image douteuse: on croit que la justice se marchande, s'achète. Bien que notre recherche indique que la majorité des négociations de plaidoyer ne revêtent pas le caractère inique habituellement accolé à la pratique, il demeure néanmoins que le principe d'apparence de justice n'est pas respecté.

\section{BIBLIOGRAPHIE}

BARREAU DU QUÉBEC (1987), Droit pénal. Cours de la formation professionnelle du Barreau du Québec, 14, Montréal, Les Éditions Yvon Blais Inc.

COMMISSION CANADIENNE SUR LA DÉTERMINATION DE LA PEINE (1987), Réformer la sentence: une approche canadienne, Ottawa, Approvisionnements et Services Canada.

COUSINEAU, F. D. et VERDUN-JONES, S. N. (1979), «Evaluating Research into Plea Bargaining in Canada and the United States; Pit Falls Facing the Policy Makers», Revue canadienne de criminologie, vol. $21, n^{\circ} 3$, p. 289-309. 
ERICSON, R. V. et BARANEK, P. M. (1982), The Ordering of Justice : A Study of Accused Persons as Dependants in the Criminal Process, Toronto, University of Toronto Press.

FEELEY, M. M. (1979), The Process is the Punishment: Handling Cases in a Lower Criminal Court, New York, Russell Sage Foundation.

FERGUSON, G. A. et ROBERTS, D. W. (1974), «Plea Bargaining: Directions for Canadian Reform", Revue du Barreau canadien, vol. 52, p. 498-576.

GRAVEL, S. et BARIL, M. (1990), La Pratique de la négociation de plaidoyer au Palais de Justice de Montréal, Montréal, Centre international de criminologie comparée, Université de Montréal.

GROSMAN, B. A. (1969), The Prosecutor. An Inquiry into the Exercise of Discretion, Toronto, University of Toronto Press.

HARTNAGEL, T. N. (1975), «Plea Negotiation in Canada», Revue canadienne de criminologie, vol. $17, \mathrm{n}^{\circ} 1$, p. $45-66$.

HEUMANN, M. (1978), Plea Bargaining: The Experiences of Prosecutors, Judges and Defence Attorneys, Chicago, University of Chicago Press.

HOGARTH, J. (1971), Sentencing as a Human Process, Toronto, University of Toronto Press.

KLEIN, J. F. (1976), Let's Make a Deal : Negotiating Justice, Lexington, Mass., Lexington Books.

MATHER, L. M. (1979), Plea Bargaining or Trial? The Process of Criminal Case Disposition, Lexington, Mass., Lexington Books.

MAYNARD, D. W. (1984), Inside Plea Bargaining : The Language of Negotiation, New York, Plenum Press.

NARDULLI, P. F., FLEMMING, R. B. et EISENSTEIN, J. (1985), «Criminal Courts and Bureaucratic Justice: Concessions and Consensus in the Guilty Plea Process», The Journal of Criminal Law and Criminology, vol. 76, $\mathrm{n}^{\circ}$ 4, p. 1103-1131.

POIRIER, R. (1986), Des pratiques de travail des avocats de la défense dans $l^{\circ}$ organisation pénale, mémoire de maîtrise ès sciences (criminologie), Université de Montréal, École de criminologie.

PROCUREUR GÉNÉRAL DU QUÉBEC (1988), Guide à l'intention des substituts du Procureur général, document de travail.

SOLOMON, P. H. Jr. (1983), Criminal Justice Policy, From Research to Reform, Toronto, Butterworths.

SUDNOW, D. (1965), «Normal Crimes», in E. Rubington et M. S. Weinberg (éd.), Deviance: The Interactionist Perspective, New York, MacMillan Co. (1973), p. 174-186. 